\title{
XPS Spectra of Copper and Nickel Biuret Complexes - Observations of Intense Satellite Structure in the 2P Spectrum of a Copper(III) System
}

\author{
Dennis G. Brown and Ulrich Weser \\ Anorganische Biochemie, Physiologisch-Chemisches Institut der Universität Tübingen, \\ Hoppe-Seyler-Straße 1, D-7400 Tübingen 1, F.R.G.
}

Z. Naturforsch. 34b, 989-994 (1979); received March 30, 1979

Copper, Nickel, Photoelectron Spectra, Satellite

\begin{abstract}
A series of copper and nickel biuret complexes has been studied by X-ray photoelectron spectroscopy (XPS). The results include one of the first reports of the XPS spectrum of a copper(III) complex. Significantly, this diamagnetic complex exhibits intense satellite structure in both the $\mathrm{Cu} 2 \mathrm{P}_{3 / 2}$ and $\mathrm{Cu} 2 \mathrm{P}_{1 / 2}$ regions. The isoelectronic nickel(II) complex shows no comparable satellite structure.
\end{abstract}

\section{Introduction}

It has been demonstrated rather clearly that the X-ray photoelectron spectroscopy (XPS) of copper complexes is a useful means of distinguishing between the copper(I), $3 \mathrm{~d}^{10}$, and copper(II), $3 \mathrm{~d}^{9}$, electronic states [1-3]. Thus, the XPS of copper(I) complexes exhibits a symmetrical main peak in either the $2 \mathrm{P}_{3 / 2}$ or $2 \mathrm{P}_{1 / 2}$ region with no evidence of a shake-up satellite at higher binding energy. Copper(II), on the other hand, exhibits, in addition to the main peak, one or two satellite peaks at $5-10 \mathrm{eV}$ higher binding energy from the main peak. Even in copper(I)-copper(II) mixed valence compounds the two oxidation states can be resolved by XPS [4]. In most instances the satellite is an asymmetrical peak due to two or more overlapping but unresolved satellite peaks. In some complexes, however, two well separated and well defined satellites are observed. We have shown that this occurs when the copper is bound to carbonyl oxygen atoms [1]. One of us has discussed the possible applications of copper XPS to the study of biologically relevant ligand systems and proposed to use this technique for the study of biochemically active copper $[1,2,4,5]$.

Because of the recent interest in the possibility of copper(III) in biological systems [6-8], it was of interest to obtain XPS spectra on a well defined

Reprint requests to Prof. D. Brown, Department of Chemistry, University of Idaho, Moscow, Idaho, U.S.A., or to Prof. U. Weser, Anorganische Biochemie, Physiologisch-Chemisches Institut der Universität Tübingen, Hoppe-Seyler-Straße 1, D-7400 Tübingen 1, F.R.G.

$0340-5087 / 79 / 0700-0989 / \$ 01.00 / 0$
copper(III)complex to ascertain whether this technique could be of potential use in observing copper(III) in a copper dependent enzyme system. In addition, when this work was begun, no copper(III) XPS studies had appeared in the literature and the possibility of characterizing this oxidation state by XPS was of inherent interest. During the course of this work, the $2 \mathrm{P}_{1 / 2}$ and $2 \mathrm{P}_{3 / 2}$ binding energy values of a copper(III) macrocycle were reported [9]. A number of copper(III) systems are known in inorganic chemistry [9-14]. Many of these are somewhat unstable, decomposing readily to copper(II). For an initial investigation of copper(III) XPS it was very important to deal with as stable a complex as possible. In addition, we desired to have available the analogous copper(II) complex with which to compare XPS parameters. For this work, therefore, the bis biureto (-HNCONHCONH- $=$ bi) complexes of copper were chosen. Both the copper(III) species, $\mathrm{KCu}(\mathrm{bi})_{2}$, and the copper(II) species, $\mathrm{K}_{2} \mathrm{Cu}(\mathrm{bi})_{2}$, are known and well charaterized $[10,15]$. An additional advantage of this ligand system is the fact that the analogous nickel(II) and nickel(III) complexes are available. This is especially useful in that the copper(III) and nickel(II) compounds are isoelectronic and should provide an interesting comparison. In addition, the series of complexes allows a comparison of the metal electronic configurations $\mathrm{d}^{7}$, $d^{8}$, and $d^{9}$ with the same ligand environments.

We report here the XPS spectra of the above mentioned series of copper and nickel biureto complexes. The observation of an intense satellite for the copper(III) compound $\mathrm{KCu}(\mathrm{bi})_{2}$ while such satellites are not present in a copper(III) macrocycle $[9 \mathrm{a}]$ nor in the isoelectronic nickel(II) species 
raises questions about the general interpretation of shake-up satellites in these transition metals.

\section{Experimental}

\section{$X$-ray photoelectron spectroscopy}

$\mathrm{X}$-ray photoelectron spectra were obtained with a Varian V-IEE 15 high-resolution electron spectrometer equipped with a $620-\mathrm{L}$ online computer $(8 \mathrm{~K})$. During the course of the measurement the sample was maintained at approximately $100 \mathrm{~K}$ by cooling with liquid nitrogen. This has proven satisfactory to minimize decomposition reactions caused by $\mathrm{X}$-ray-irradiation. The samples were run as finely ground powders dusted onto the backing of a onesided adhesive Cellotape (Scotch Tape, 3M Co.) which was attached to the cylindrical sample holder. The spectra were standardized with the C IS aliphatic hydrocarbon line for which a binding energy of $284.0 \mathrm{eV}$ was assigned [16]. All spectra were time averaged and smoothed using a box car function.

\section{Compounds}

Reagent grade copper(II) acetate monohydrate, nickel(II) acetate monohydrate, and biuret were from Merck, Darmstadt. $\mathrm{K}_{2} \mathrm{Cu}(\mathrm{bi})_{2}$ and $\mathrm{K}_{2} \mathrm{Ni}(\mathrm{bi})_{2}$ were prepared according to the method of Freeman $[15] . \mathrm{KCu}(\mathrm{bi})_{2}$ and $\mathrm{KNi}(\mathrm{bi})_{2}$ were prepared according to the method of Bour et al., by $\mathrm{S}_{2} \mathrm{O}_{8}=$ oxidation of solutions of the corresponding $\mathrm{K}_{2} \mathrm{M}(\mathrm{bi})_{2}$ compounds [10].

\section{Results and Discussion}

The compounds studied here are all square planar with nitrogen donor atoms. The $\mathrm{X}$-ray crystal structure for $\mathrm{K}_{2} \mathrm{Cu}(\mathrm{bi})_{2} \cdot 4 \mathrm{H}_{2} \mathrm{O}$ demonstrates this unambiguously for the copper(II) complex [15]. Kedzia et al., have shown that the nickel(II) compound has the same structure as the copper(II) species [17]. Bour et al. have advanced a number of arguments to indicate that the copper(III) and nickel(III) species are also square planar [10]. The fact that the copper(III) and nickel(II) species are isoelectronic argues strongly for similar coordination geometries. Low spin $d^{8}$ complexes are virtually always square planar. Magnetic measurements on the nickel(II), nickel(III), and copper(III) complexes reveals that they are all low spin, as expected for the strong equatorial ligand field arising from the deprotonated nitrogen donor atoms [10]. All this evidence indicates rather unambiguously that the complexes have nearly identical coordination environments. The simple one-electron $d$ orbital configurations thus are as follows.

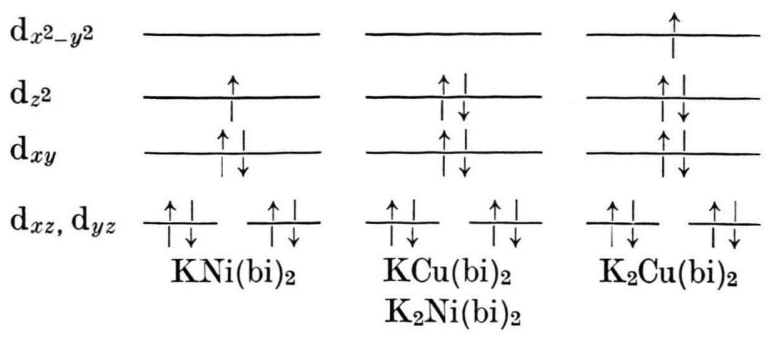

It is significant for the discussion which follows to note that the nickel(II) and copper(III) are isoelectronic and, with respect to the coordination environment, nearly isostructural.

The $2 \mathrm{P}_{3 / 2}$ XPS spectra for the compounds are shown in Figs. 1 and 2. The significant features are as follows. The nickel(II) compound, $\mathrm{K}_{2} \mathrm{Ni}(\mathrm{bi})_{2}$

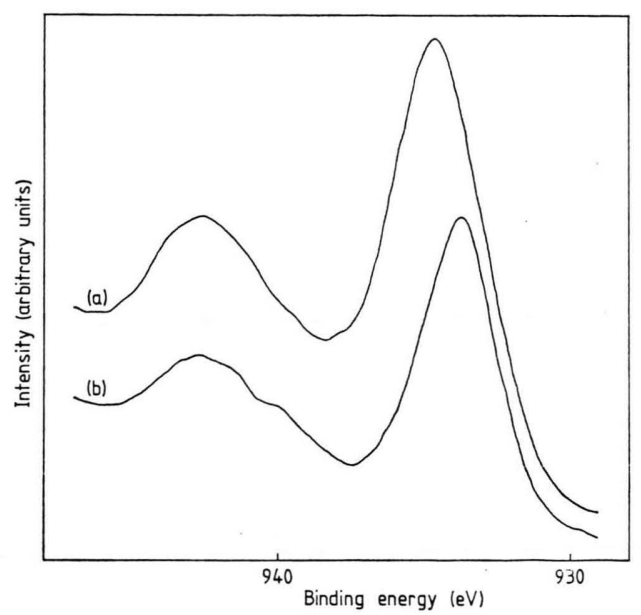

Fig. 1. $2 \mathrm{P}_{3 / 2} \mathrm{XPS}$ spectra for (a) $\mathrm{KCu}(\mathrm{bi})_{2}$ and (b) $\mathrm{K}_{2} \mathrm{Cu}(\mathrm{bi})_{2}$. For further information see text.

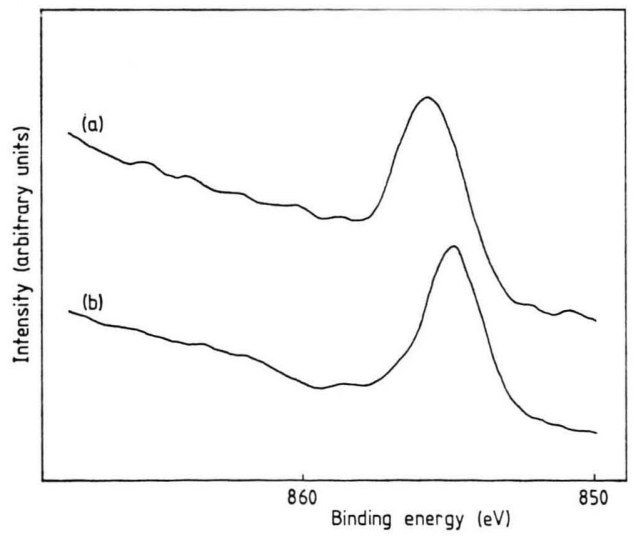

Fig. 2. $2 \mathrm{P}_{3 / 2} \mathrm{XPS}$ spectra for (a) $\mathrm{KNi}(\mathrm{bi})_{2}$ and (b) $\mathrm{K}_{2} \mathrm{Ni}(\mathrm{bi})_{2}$. For further information see text. 
exhibits only a main peak with no satellite(s) of appreciable intensity as has been observed for a number of low spin nickel(II) systems. The nickel(III) complex also demonstrates only a main peak with, at best, very weak satellite structure. The copper(II) compound shows a strong, somewhat asymmetric satellite peak at $8.7 \mathrm{eV}$ higher binding energy than the main peak. Both the position and shape of this satellite are quite normal for copper(II) surrounded by four nitrogen ligand atoms. The copper(III) spectrum, the second reported for a copper(III) complex, exhibits a strong and rather symmetrical peak at $7.9 \mathrm{eV}$ higher binding energy from the main peak. The following observations are pertinent.

1) The satellite peak is not due to a reduction to copper(II) in the X-ray beam as has been reported for a variety of systems [2, 18-21]. The spectrum was recorded with the sample at approximately $100 \mathrm{~K}$. Our experience with this sample handling in our instrument indicates that in those cases where some decomposition occurs at ambient temperature, it is completely prevented at the lower temperature. There is, in fact, no dependence of spectral features with length of exposure to the $\mathrm{X}$-ray beam at either low temperatures or room temperature, as might be expected, if decomposition were occurring. Even at ambient temperatures this compound appears to be stable under conditions of spectral measurement.

2) We have also obtained the spectrum of the analogous copper(II) compound. If the origin of the satellite in the copper(III) spectrum were due, in fact, to reduction to copper(II), the spectrum known for the copper(II) complex should be obtained. On the contrary, the fact that the spectral parameters for the copper(II) and copper(III) compounds are substantially different indicates that they arise from different species.

The values for the binding energies and other spectral parameters for the compounds studied are given in Table I. Several points deserve comment. The metal $2 \mathrm{P}_{3 / 2}$ binding energies (main peak) for the +3 oxidation states is, as expected, higher than for the +2 oxidation states. Significantly, however, the copper binding energy for this copper(III) complex is lower than is found for a number of copper(II) systems. For example, the $2 \mathrm{P}_{3 / 2}$ binding energy for $\mathrm{KCu}(\mathrm{bi})_{2}(934.5 \mathrm{eV})$ is lower than that for $\mathrm{CuF}_{2}(938.5 \mathrm{eV}), \mathrm{CuO}(935.7 \mathrm{eV}), \mathrm{Cu}$ (oxalate) $(935.4 \mathrm{eV})$, and $\mathrm{CuCl}_{2}(935.1 \mathrm{eV})$ [1]. This is undoubtedly due to the fact that the deprotonated nitrogen atoms are very good electron donors, capable apparently of donating enough electron density to the formally copper(III) center that the actual charge on copper is comparable to that found in many copper(II) systems. This, of course, is the fundamental reason that this particular ligand system, as well as others which have deprotonated nitrogen donor atoms, is capable of stabilizing the copper(III) oxidation state. A similar situation holds for the nickel(III) complex. Frost et al. have determined an empirical correlation between actual charge on a central copper ion versus observed binding energy for a series of copper(II) complexes [2]. If this qualitative correlation holds for the systems reported here, then a difference of $1 \mathrm{eV}$ in the binding energies for the copper(II) and copper(III) systems corresponds to 0.3-0.4 units of actual charge.

With respect to satellite structure, the copper(II) complex, $\mathrm{K}_{2} \mathrm{Cu}(\mathrm{bi})_{2}$, shows the normal satellite at higher binding energy. The satellite intensity amounts to $30 \%$ of the total signal intensity. The satellite, as is often the case, is composed of 2 components which lie at about $8.7 \mathrm{eV}$ and $6.1 \mathrm{eV}$ to higher binding energy from the $2 \mathrm{P}_{3 / 2}$ main peak. The satellite intensity for the copper(III) case corresponds to $25 \%$ of the total signal intensity. The copper(III) satellite always appears to be more symmetrical than that for the copper(II) compound

Table I. Electron binding energies.

\begin{tabular}{|c|c|c|c|c|c|c|}
\hline Compound & N 1s (eV) & $\mathrm{C} 1 \mathrm{~s}(\mathrm{eV})$ & $\begin{array}{l}\text { M } 2 \mathrm{P}_{1 ! 2}(\mathrm{eV}) \\
\text { Satellite }\end{array}$ & Main peak & $\begin{array}{l}\text { M } 2 \mathrm{P}_{3 / 2}(\mathrm{eV}) \\
\text { Satellite }\end{array}$ & Main peak \\
\hline $\mathrm{K}_{2} \mathrm{Cu}(\mathrm{bi})_{2}$ & 399.0 & 287.8 & 962.2 & 953.8 & 942.4 & 933.7 \\
\hline $\mathrm{KCu}(\mathrm{bi})_{2}$ & 398.8 & 287.8 & 962.1 & 954.8 & 942.4 & 934.7 \\
\hline $\mathrm{K}_{2} \mathrm{Ni}(\mathrm{bi})_{2}$ & 398.7 & 287.5 & - & 871.9 & - & 854.8 \\
\hline $\mathrm{KNi}(\mathrm{bi})_{\mathbf{2}}$ & 398.7 & 287.6 & - & 872.9 & - & 855.7 \\
\hline $\mathrm{biH}_{2}$ & 399.3 & 288.3 & & & & \\
\hline
\end{tabular}


and it lies somewhat nearer the main peak at $7.9 \mathrm{eV}$ to higher binding energy. Another characteristic difference between the spectra of the two copper systems is the width of the main peak. The full width at half maximum intensity for the $2 \mathrm{P}_{3 / 2}$ peak of the copper(II) system is about $3.0 \mathrm{eV}$ (an average value from several spectra). This value is quite normal for copper(II). On the other hand a corresponding line width for the copper(III) complex is $4.2 \mathrm{eV}$ (also an average from several spectra). In the nickel complexes, the nickel(III) compound appears to have a slightly greater line width than does the nickel(II) but the difference is not nearly as great as for the two copper compounds.

For some first row transition metals, the difference in binding energy between the $2 \mathrm{P}_{3 / 2}$ and $2 \mathrm{P}_{1 / 2}$ main peak is characteristic of the spin state. Thus, Frost et al., have reported a number of cobalt(II) and cobalt(III) complexes in which the $2 \mathrm{P}_{1 / 2}-2 \mathrm{P}_{3 / 2}$ main peak energy separation was consistently approximately $1 \mathrm{eV}$ larger for the paramagnetic cobalt(II) than for the diamagnetic cobalt(III) compound [22a]. Briggs and Gibson have reported similar information $[22 \mathrm{~b}]$. In the same way, high spin nickel(II) compounds show about $0.5 \mathrm{eV}$ larger $2 \mathrm{P}_{1 / 2}-2 \mathrm{P}_{3 / 2}$ separation than do diamagnetic low spin nickel(II) compounds [23]. A comparison of a large number of copper(I) and copper(II) complexes, however, reveals no measurable difference in the $2 \mathrm{P}_{1 / 2}-2 \mathrm{P}_{3 / 2}$ separation $[1,2]$. For the copper(III) complex reported here a $2 \mathrm{P}_{1 / 2}-2 \mathrm{P}_{3 / 2}$ separation of $20.1 \mathrm{eV}$ is found, again indistinguishable from copper(II) and copper(I). Thus, all three copper oxidation states appear to have the same $2 \mathrm{P}_{1 / 2}-2 \mathrm{P}_{3 / 2}$ separation.

With respect to the XPS spectra of the other atoms, very little information can be obtained which sheds light on the electronic structures of the complexes in question. Some of these parameters have also been reported recently by Yoshida $e t a l$. [24] on a number of biureto systems. The nitrogen IS binding energies for all the complexes are lower than for pure biuret. This is not surprising in view of the fact that the complexes contain deprotonated terminal nitrogen atoms. The differences in nitrogen binding energies among the series of complexes are small enough so as to be considered negligible. It is somewhat surprising that the nitrogen atoms in the complexes with the +3 oxidation state do not show measurably higher binding energies. There are, of course, in all the complexes two types of nitrogen atoms. The binding energies given in Table I are simply the observed maxima in the N IS peaks. This peak is, however, consistently broader $(3.0-3.3 \mathrm{eV})$ than the peak width at half maximum height normally observed in compounds with only one type of nitrogen atom. Yoshida et al., have reported separate values for the two nitrogens in certain biureto complexes [24], but we find that substantially different values can be obtained which depend upon the assumptions made in carrying out the resolution into 2 peaks. We have, therefore, only reported the binding energies for the maximum intensity for each peak. In pure biuret, as well as in the complexes, the carbonyl carbon atoms have a binding energy which occurs high enough so that it is well resolved from the carbon reference signal. It is also observed that the ligand carbon binding energies are lower in the complexes than in pure biuret, again an effect of the double deprotonation of the ligand. As is the case for nitrogen, the carbon binding energies are virtually the same regardless of the metal oxidation state.

Although current instrumentation is at best marginal for XPS investigation of copper sites in biological molecules, future improvements in signal intensity should lead to more wide spread use of XPS in this area. It appears, however, that while XPS will allow a convenient means of distinguishing between copper(I) and copper(II), one cannot anticipate distinguishing between copper(II) and copper(III) by this technique. From the present results neither the binding energy nor the satellite pattern clearly distinguish these two oxidation states. This is especially true because the chemical environment in which one will find copper(III), particularly in an aqueous system, will invariably be one in which the copper is bound to deprotonated nitrogen ligand atoms [7-9]. This ligand environment allows considerable electron donation to the copper (which is the cause of the stability of this oxidation state) with a resulting binding energy lying in the range often observed for copper(II). It may be possible that the line width of the main copper signal could be used to distinguish between these two oxidation states, but several copper(III) systems will have to be investigated before any general conclusions can be drawn.

The most fundamental and interesting question arising from this work is that of the satellite struc- 
ture of the copper(III) bis biureto complex. Some workers have implied that the presence of the unpaired electron in copper(II) is responsible for the presence of the copper satellites and a correlation between copper electron spin density and satellite intensity has even been reported [25]. The presence or absence of a satellite, however, appears indeed not to be a direct result of the presence or absence of unpaired electrons. The absence of a satellite in copper(I), for example, is simply due to the fact that the $d$ orbitals are completely filled so that empty antibonding orbitals are not available for charge transfer [31]. The copper(III) system reported here is diamagnetic yet a satellite is observed. A substantial amount of theoretical work has been reported aimed at understanding the satellite structure in transition metal complexes, especially those observed for copper(II) [3 b, 26-34]. If one adopts, for example, the formalism of Larsson [31-34], then it appears that there is no inherent reason to expect drastically different behaviour for copper(II) and copper(III). The charge transfer mechanism which this author suggests for copper(II) would be equally valid for copper(III). The primary difference in the two cases would be that the latter has two $A_{1 g}$ spin orbitals through which charge transfer could occur, rather than the one available in copper(II). Since, in general, we observe rather similar binding energies, especially for the ligand atoms, for both the copper(II) and copper(III) complexes, one would probably not expect drastically different metal and ligand contributions to the pertinent m.o.'s in the two systems. Thus, within the framework of Larsson's formalism, we conclude that the spectral properties observed for $\mathrm{KCu}(\mathrm{bi})_{2}$ are not inconsistent with the theoretical discussions which have been offered for copper satellite structure.
In this work we have clearly obtained a well defined satellite for a copper(III) complex. It is puzzling that a recently reported copper(III) macrocycle complex, shows no satellite structure [9a]. Both systems seem clearly to be copper(III) based on a variety of physicochemical measurements. In addition, the direct comparison of isoelectronic $\mathrm{KCu}(\mathrm{bi})_{2}$ and $\mathrm{K}_{2} \mathrm{Ni}(\mathrm{bi})_{2}$ is interesting. The former compound shows satellite structure while the latter does not [36]. Using Larsson's formalism and results of molecular orbital or X-alpha calculations of the relative metal and ligand contributions to the $B_{1 g}$ and $\mathrm{B}_{1 \mathrm{~g}}^{*}$ molecular orbitals, one can conclude that the satellite intensity for low spin nickel(II) should be at least equal to or greater than the corresponding satellite in the isoelectronic copper(III) complex [35]. (The possibility remains, of course, that different mechanisms give rise to satellites in copper and nickel, in which case no comparisons between these systems could be made.) It appears that the great difference in satellite intensity between these two isoelectronic systems remains theoretically unexplained.

It is possible that the presence of satellite structure in $\mathrm{KCu}(\mathrm{bi})_{2}$ or its absence in the copper(III) macrocycle complex of Keyes et al. [9a], is an anomaly. However, to date only two copper(III) XPS spectra have been reported. More copper(III) systems will have to be studied by this technique before any general conclusions can be drawn.

D. G. B. is grateful to the Alexander von Humboldt Foundation for the award of a research fellowship for 1978-79. U. W. is a recipient of a grant-in-aid (DFG 401/14). Thanks go to Karin Rupp for skillful technical assistance.
[1] H. Rupp and U. Weser, Biochim. Biophys. Acta 446, 151 (1976).

[2] D. C. Frost, A. Ishitani, and C. A. McDowell, Mol. Phys. 24, 861 (1972).

[3] a) T. Novakov, Phys. Rev. B 3, 2693 (1971); b) K.S. Kim, J. Electron Spectrosc. and Relat. Phenom. 3, 217 (1974);

c) A. Rosencwaig and G. K. Wertheim, J. Electron Spectrosc. and Relat. Phenom. 1, 493 (1972/73);

d) R. F. Roberts, J. Electron Spectrosc. and Relat. Phenom. 4, 273 (1974);

e) P. E. Larson, J. Electron Spectrosc. and Relat. Phenom. 4, 213 (1974); f) D. C. Frost, C. A. McDowell, and R. L. Trapping, J. Electron Spectrosc. and Relat. Phenom. 6, 374, and 7, 297 (1974).

[4] H. Rupp and U. Weser, Bioinorg. Chem. 6, 45 (1976).

[5] a) G. Jung, M. Ottnad, W. Bohnenkamp, and U. Weser, FEBS Lett. 25, 346 (1972);

b) G. Jung, M. Ottnad, W. Bohnenkamp, W. Bremser, and U. Weser, Biochim. Biophys. Acta 295, 77 (1973);

c) U. Weser, F. Donay, and H. Rupp, FEBS Lett. 32, 171 (1973);

d) G. Sokolowski, W. Pilz, and U. Weser, FEBS

Lett. 48, 222 (1974); 
e) R. Prinz and U. Weser, FEBS Lett. 54, 224 (1975);

f) W. Thomas and U. Weser, Hoppe-Seyler's Z. Physiol. Chem. 358, 47 (1977);

g) U. Weser, G. Sokolowski, and W. Pilz, J. Electron Spectrosc. and Relat. Phenom. 10, 429 (1977).

[6] a) G. A. Hamilton, R. D. Libby, and C. R. Hartzell, Biochem. Biophys. Res. Commun. 55, 333 (1976);

b) G. R. Dyrkacz, R. D. Libby, and G. A. Hamilton, J. Am. Chem. Soc. 98, 626 (1976);

c) G. A. Hamilton, P. K. Adolf, J. de Jersey, G. C. DuBois, G. D. Dyrkacz, and R. D. Libby, J. Am. Chem. Soc. 100, 1899 (1978).

[7] a) D.W. Margerum, K. L. Chellappa, F. P. Bossu, and G. L. Burce, J. Am. Chem. Soc. 97, 6894 (1975);

b) F. P. Bossu, K. L. Chellappa, and D. W. Margerum, J. Am. Chem. Soc. 99, 2195 (1977).

[8] P. Stevens, J. M. Waldeck, J. Strohl, and R. Nakon, J. Am. Chem. Soc. 100, 3632 (1978).

[9] a) W. E. Keyes, W. E. Schwartz, Jr., and T. M. Loehr, Inorg. Chem. 17, 3316 (1978);

b) W. E. Keyes, J. B. R. Dunn, and T. M. Loehr, J. Am. Chem. Soc. 99, 4527 (1977).

[10] a) J. J. Bour and J. J. Steggerda, J. Chem. Soc. Chem. Commun. 1967, 85;

b) J. J. Bour, P. J. Birker, and J. J. Steggerda, Inorg. Chem. 10, 1202 (1971).

[11] R. Scholder and U. Vaelskow, Z. Anorg. Chem. 266, 256 (1951).

[12] D. C. Olson and J. Vasilevskis, Inorg. Chem. 10, 463 (1971).

[13] A. R. Hendrickson, R. L. Martin, and N. M. Rohde, Inorg. Chem. 15, 2115 (1976).

[14] P. J. M. W. L. Birker, Inorg. Chem. 16, 2478 (1977).

[15] H. C. Freeman, J. E. W. L. Smith, and J. C. Taylor, Acta Crystallogr. 14, 407 (1961).

[16] K. Siegbahn, C. Nordling, A. Fahlman, R. Nordberg, K. Hamrin, J. Hedman, G. Johansson, T. Bergmark, S. E. Karlsson, I. Lindgren, and B. Lindberg, ESCA, Atomic, Molecular and Solid State Structures Studied by Means of Electron Spectroscopy, Almqvist and Wiksells, Uppsala 1967.
[17] B. B. Kedzia, P. X. Armendarez, and K. Nakamoto, J. Inorg. Nucl. Chem. 30, 849 (1968).

[18] B. Wallbank, C. E. Johnson, and I. G. Main, J. Electron Spectrosc. and Relat. Phenom. 4, 263 (1974).

[19] P. Burrough, A. Hamnet, J. McGilp, and A. Orchard, J. Chem. Soc. Faraday Trans. II 1975, 177.

[20] M. Batista-Leal, J. E. Lester, and C. A. Lucchesi, J. Electron Spectrosc. and Relat. Phenom. 11, 333 (1977).

[21] R. G. Copperwaith and J. Lloyd, J. Electron Spectrosc. and Relat. Phenom. 14, 159 (1978).

[22] a) D. C. Frost, C. A. McDowell, and I. S. Woolsey, Chem. Phys. Lett. 17, 320 (1972);

b) D. Briggs and V. A. Gibson, Chem. Phys. Lett. 25, 493 (1972).

[23] C. K. Jorgensen, Chimia 25, 213 (1971).

[24] T. Yoshida, K. Yamasaki, and S. Sawada, Bull. Chem. Soc. Jpn. 51, 1561 (1978).

[25] M. S. Ioffe and Y. G. Borod'ko, J. Electron Spectrosc. and Relat. Phenom. 11, 235 (1977).

[26] A. Rosencwaig, G. K. Wertheim, and H. J. Guggenheim, Phys. Rev. Lett. 27, 479 (1971).

[27] A. Kotani and Y. Toyozawa, J. Phys. Soc. Jpn. 37, 573 (1974).

[28] S. Asada and S. J. Sugano, J. Phys. Soc. Jpn. 41, 1291 (1976).

[29] G. A. Vernon, G. Stucky, and T. A. Carlson, Inorg. Chem. 15, 278 (1976).

[30] C. K. Jorgensen, in P. O. Löwdin (ed.): Advances in Quantum Chemistry, Vol. 8, pp. 137-182, Academic Press, New York 1974.

[31] S. Larsson, Chem. Phys. Lett. 32, 401 (1975).

[32] S. Larsson, Chem. Phys. Lett. 40, 362 (1976).

[33] S. Larsson, Physica Scripta 16, 378 (1977).

[34] S. Larsson and E. Odeblad, Physica Scripta 16, 381 (1977).

[35] See ref. 31 and references cited therein.

[36] It appears actually from several of our $\mathrm{Ni} 2 \mathrm{P}_{3 / 2}$ spectra for $\mathrm{K}_{2} \mathrm{Ni}(\mathrm{bi})_{2}$ that there may be very weak satellites at approximately 6 and $8.5 \mathrm{eV}$ above the main peak. However, an upper limit on the satellite intensity would be perhaps $2 \%$ of the total signal intensity. 